\title{
Adipose vascular endothelial growth factor $B$ is a major regulator of energy metabolism
}

\author{
Yang Chen 1,*, Mingyue Zhao1,*, Chenhao Wang1, Huaizhen Wen1, Yuntao Zhang1, Mingxu Lu¹, Salah Adlat', \\ Tingting Zheng1, Mingjiao Zhang1, Dan Li1, Xiaodan Lu1', Mengwei Guo1, Hongyu Chen', Luqing Zhang1,2, \\ Xuechao Feng1,2 and Yaowu Zheng1,2
}

1Transgenic Research Center, Northeast Normal University, Changchun, China

${ }^{2}$ Key Laboratory of Molecular Epigenetics of Ministry of Education, Northeast Normal University, Changchun, China

Correspondence should be addressed to Y Zheng: zhengyw442@nenu.edu.cn

*(Y Chen and M Zhao contributed equally to this work)

\begin{abstract}
Excessive fat accumulation causes obesity and many diseases. Previous study demonstrates VEGFB universal knockout induces obese phenotypes including expansion of white adipose tissue, whitening of brown adipose tissue, increase of fat accumulation and reduction in energy consumption. However, roles of VEGFB in adipose tissues are not clear. In this study, we have generated a mouse model with adipose-specific VEGFB repression using CRISPR/dCas9 system (Vegfb ${ }^{\text {AdipoDown }}$ ) and investigated the roles of VEGFB in adipose development and energy metabolism. VEGFB repression induced significant changes in adipose tissue structure and function. VegfbadipoDown mice have larger body sizes, larger volume of white adipose tissues than its wild type littermates. Adipose-specific VEGFB repression induced morphological and functional transformation of adipose tissues toward white adipose for energy storage. Metabolic processes are broadly changed in VegfbAdipoDown adipose tissues including carbohydrate metabolism, lipid metabolism, nucleotide metabolism and amino acid metabolism. We have demonstrated that adipose VEGFB repression can recapitulate most of the phenotypes of the whole body VEGFB knockout mouse. Intriguingly, approximately 50\% VEGFB repression in adipose tissues can almost completely mimic the effects of universal Vegfb deletion, suggesting adipose VEGFB is a major regulator of energy metabolism and may be important in prevention and treatment of obesity.
\end{abstract}

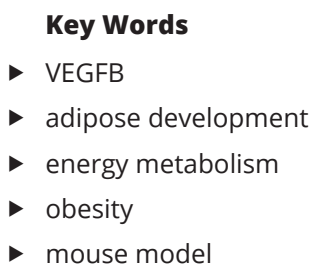

Journal of Endocrinology (2020) 244, 511-521

\section{Introduction}

Obesity, an epidemic and chronic disease induced by excessive energy storage and adipose expansion, affects millions of people (Kopelman 2000, WHO Expert Consultation 2004, James 2008). An imbalanced energy intake and expenditure leads to the excessive adipose expansion (Cai et al. 2010). This excessive adipose expansion is associated with hypertension, type 2 diabetes, atherosclerosis, heart disease, infertility, stroke and certain types of malignancies (Madsen et al. 2010, Castro et al. 2014, Luna-Luna et al. 2015, Mission et al. 2015, Cedikova et al. 2016). Adipose tissues are classified as white adipose tissues (WATs) and brown adipose tissue 
(BAT). White adipocytes develop from Myf5-negative mesenchymal precursor cells (Cinti 2008, Rodeheffer et al. 2008) and look spherical in morphology. White adipocytes are filled with a single, large lipid droplet, small cytoplasm and few mitochondria on the edge of the cell. WAT acts as an energy storage organ in form of triglycerides. At the same time, WAT is an important secretory organ and produces leptin, adiponectin, resistin and growth factors including vascular endothelial growth factors (VEGFs) (Mick et al. 2002, Fain et al. 2004, Kershaw \& Flier 2004). Brown adipocytes, in contrast, originate from precursors that express Myf5 (Kopelman 2000) and contain a huge number of mitochondria and multiple small lipid droplets for efficient energy use. BAT is rich in vasculature and expresses high levels of uncoupling protein 1 (UCP-1) and can generate heat quickly from lipid oxidation through uncoupling respiratory chain (Richard et al. 2010, Richard \& Picard 2011, Esteve Ràfols 2014, Shimizu $\&$ Walsh 2015). BAT function is critical in maintaining body temperature and body weight. An intermediate adipose tissue is also present that is called beige or brownlike adipose tissues (bATs). Beige adipocyte has mixed characteristics of both white and brown adipocyte. It usually contains multiple lipid droplets with variable size and is rich in mitochondria. The bAT is first identified from WAT under cold exposure or exercise (Enerbäck et al. 1997, De Matteis et al. 2013, Wang et al. 2013, Sun et al. 2015). At certain condition, bAT can develop from BAT as well (Kreuger et al. 2006). As bAT can transform energy to heat as efficiently as BAT, understanding of development and regulatory mechanism of bAT is important in fighting obesity.

VEGFB is a member of vascular endothelial growth factors (VEGFs) that regulate vasculogenesis and lymphogenesis during embryonic development and angiogenesis in adult (Kreuger et al. 2006, Stuttfeld \& Ballmer-Hofer 2009). Recently, VEGFB has been implicated in playing important roles in energy metabolism and lipid consumption in adipose tissue (Karpanen et al. 2008, Hagberg et al. 2010). Our previous study has demonstrated that knockout of VEGFB induces WAT expansion, WAT-associated gene upregulation, BAT whitening and BAT-associated gene downregulation (Jin et al. 2018). However, the contribution of adiposespecific VEGFB expression in adipose development and energy metabolism is not clear. In this study, we have generated a mouse model with adipose-specific VEGFB repression using CRISPR/dCas9 system and investigated the roles of VEGFB in adipose structure and function.

\section{Materials and methods}

\section{Animals, VEGFB-knockdown mice and genotyping}

All animal studies were approved by Institutional Animal Care and Use Committee for Animal Experimental Ethics, Committee of Northeast Normal University (IACUC/NENU). VEGFB-knockdown mouse model was constructed by CRISPR/dCas9 system. Details are provided in Supplementary Materials and methods (see section on supplementary materials given at the end of this article).

\section{Gene expression analysis and immunoblotting analysis}

Standard protocols were followed. See Supplementary Materials and methods for details.

Hematoxylin and eosin staining (H\&E), oil Red O staining, immunohistochemistry and fluorescein angiography

See Supplementary Materials and methods for details of in vitro studies.

Intraperitoneal glucose tolerance test (IPGTT), intraperitoneal insulin tolerance test (IPITT), body temperature, oxygen consumption measurement and metabolic chamber analyses

Standard protocol were provided in Supplementary Materials and methods.

\section{Primary preadipocyte culture, mature adipocytes induction and cell transfection}

Cells were cultured in DMEM/F-12 with 20\% FBS. See Supplementary Materials and methods for details.

\section{RNA-Seq and gene ontology (GO) analysis}

Genome wide gene expression was analyzed using BGISEQ-500 platform. Details are provided in Supplementary Materials and methods.

\section{Statistical analysis}

All statistical analysis for two groups was performed with SPSS software, version 20.0 (IBM). Results are expressed as mean \pm S.E.M. $P$ value $\leq 0.05$ (unpaired Student's $t$-test) 
was considered statistically significant. All graphics were prepared with GraphPad Prism 7.0 (GraphPad Software).

\section{Results}

\section{Generation of Adipose-specific VEGFB repression mouse model using CRISPR/dCas9}

To knockdown VEGFB function, two sgRNA targeting sites were selected in exon 1 region of $V e g f b$ gene. Two plasmids pU6-sgRNA1-mAdiponectin-dCas9-KRAB-T2a-GFP
(pVEGFB-sgRNA1) and pU6-sgRNA2-Adiponectin-dCas9KRAB-T2a-GFP (pVEGFB-sgRNA2) were constructed (Fig. 1A). To confirm repressive efficacy of plasmids pVEGFB-sgRNA1 and pVEGFB-sgRNA2 in VEGFBexpressing adipocytes, primary preadipocytes were isolated from sWAT of C57/BL6 mice. The primary preadipocytes were grown to confluences and then induced to mature adipocytes in culture medium containing adipocyte inducers I, II, III consecutively. To confirm successful induction, the mature adipocytes were stained with Oil Red $\mathrm{O}$ and assayed for adipose markers by quantitative
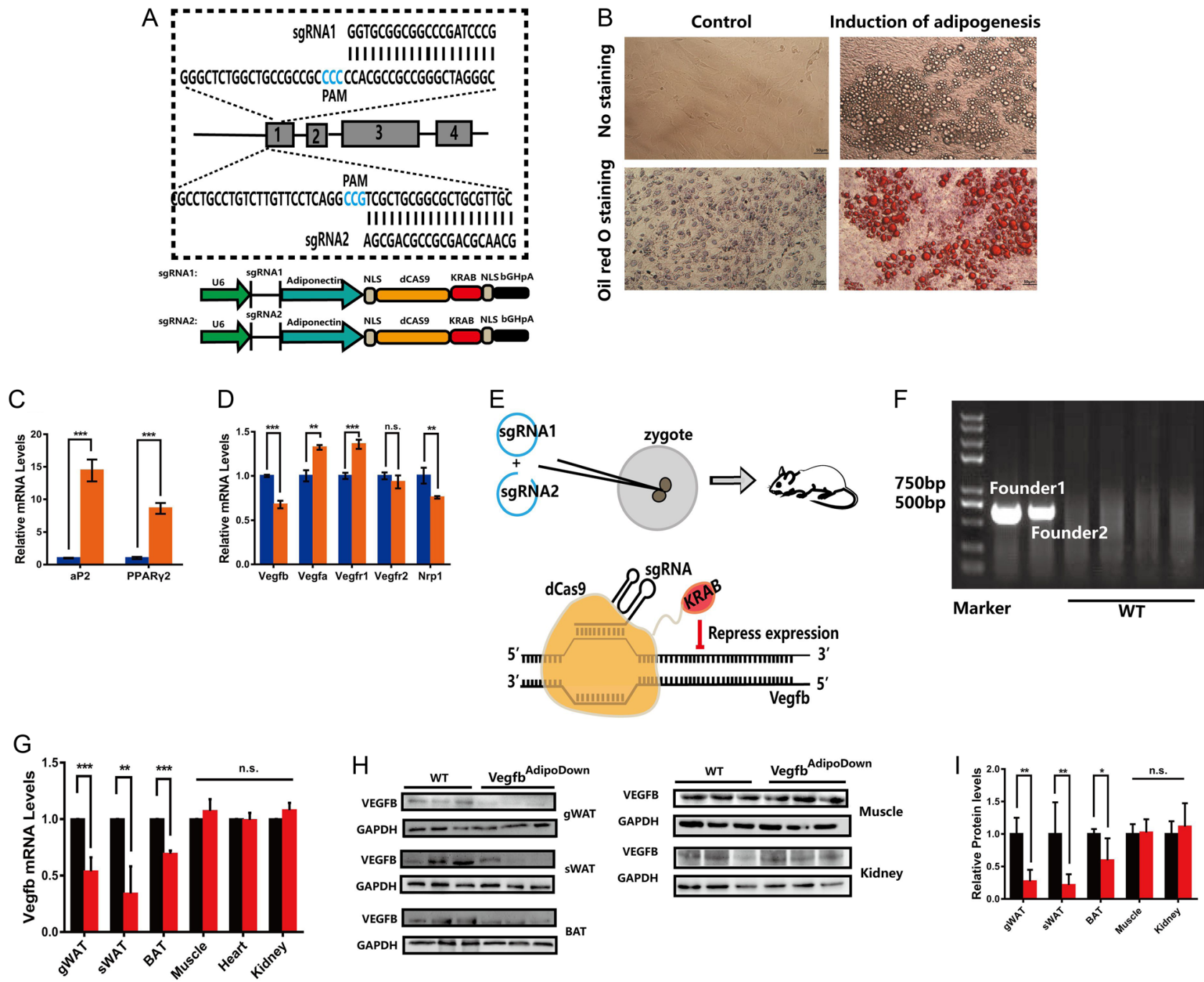

\section{Figure 1}

Generation of VEGFB repression mouse model using CRISPR/dCas9. (A) VEGFB gene structure and sgRNA target sites. Boxes and lines indicate exons and introns. (B) Culture of primary preadipocytes from C57/BL6 mice. Primary preadipocytes was induced with $0.5 \mathrm{mM} I B M X, 1 \mu \mathrm{M}$ Dex and $10 \mu \mathrm{g} / \mathrm{mL}$ insulin (top panel) and stained with Oil Red O (bottom panel). The control is uninduced preadipocytes. (C) Adipose-specific gene expression in cultured C57/BL6 primary preadipocytes by qPCR. (D) VEGFB repression analysis in transfected preadipocytes after adipokine induction. Control cell is transfected with an empty vector (Addgene \#71237) and in blue and PVEGFB-sgRNA1/2-transfected adipocytes in orange. (E) Illustration of transgenic injection of pVEGFBsgRNA1 and pVEGFB-sgRNA2 (top panel) and adipose-specific VEGFB downregulation (bottom panel). (F) Genotyping of VegfbadipoDown mice by PCR. (G) Adipose-specific VEGFB gene repression analysis by qPCR. $(\mathrm{H})$ Immunoblotting analysis of VEGFB protein. (I) Quantification of immunoblotting in $\mathrm{H}(n=3)$. VegfbAdipoDown bars in red. Data represented by mean \pm S.E.M. n.S., not significant, $\star P<0.05, * \star P<0.01, * \star \star P P<0.001$ 
real-time PCR (qPCR). Red color was readily visible after induction (Fig. 1B). Adipose-specific marker genes $a P 2$ and Ppary 2 were significantly induced (Fig. 1C). The induced mature adipocytes were then transfected with plasmids pVEGFB-sgRNA1/pVEGFB-sgRNA2 and control plasmid (3 $\mu \mathrm{g}$ each). The transfected cells were harvested $48 \mathrm{~h}$ later for qPCR. VEGFB mRNA was decreased by $32.4 \%$, whereas VEGFA mRNA was increased by $32.3 \%$. The VEGFB receptor Vegfr 1 was increased by $36.0 \%$ and $N r p 1$ decreased by $24.3 \%$ respectively. No change was found of VEGFR2, the major receptor for VEGFA and angiogenesis (Fig. 1D). Results indicate that pVEGFB-sgRNA1/2 transfection successfully induced VEGFB repression and significant changes of adipose-related genes in mature adipocytes.

To generate VEGFB-knockdown mouse model, fragments span from U6 promoter to GFP about $11 \mathrm{~kb}$ were isolated from pVEGFB-sgRNA1 and pVEGFB-sgRNA2 (Fig. 1A) and co-microinjected into C57/BL6J/DBA F1 zygotes $(2.5 \mathrm{ng} / \mu \mathrm{L}$ each) (Fig. 1E). Transgenic founders with expression of sgRNAs and dCAS9-KRAB fusion proteins were identified by genomic PCR and qPCR (Fig. 1F). VEGFB mRNA expression levels of gonadal WAT (gWAT), s.c. WAT (sWAT), interscapular BAT (BAT), skeletal muscle, heart and kidney were analyzed by qPCR (Fig. 1G) and protein levels by immunoblotting (Fig. 1H). VEGFB mRNA was found decreased significantly by $46.2 \%$ in gWAT, $65.8 \%$ in sWAT and $30.6 \%$ in BAT (Fig. 1I). However, there were no significant changes of VEGFB mRNA expression in other tissues including skeletal muscle, heart and kidney. There is a similar decrease of VEGFB protein by immunoblotting assay (Fig. $1 \mathrm{H}$ and I). VEGFB protein showed a $72.7 \%$ decrease in gWAT, 78.3\% in sWAT and 40\% in BAT (Fig. 1I).

\section{Adipose-specific VEGFB repression leads to increased energy storage}

Mice with adipose-specific VEGFB repression (VegfbAdipoDown) had bigger body size than WT mice in the same litters (Fig. 2A). Volumes of gWAT and sWAT are significantly bigger and appear whiter. The color of BAT looks brighter as well (Fig. 2B), a phenotype of adipose whitening. Blood vessel density was decreased by $52.8 \%$ in gWAT (Fig. $2 \mathrm{C}$ and $\mathrm{D})$ measured with cardiac perfusion of dextranFITC and confirmed by immunohistochemistry with CD31 (Supplementary Fig. 2A and B). The mRNA levels of hypoxia-inducible transcription factor Hif-1a was significantly decreased by $38.3 \%$ in gWAT, $39.3 \%$ in sWAT and $29.4 \%$ in BAT (Fig. 2E), indicating VEGFB knockdown did not induce oxygen stress due to reduced mitochondrial activity and lipid metabolism. The average food intakes, food efficiency and body weight gain from week 5 to week 15 were not significantly changed in Vegfb ${ }^{\text {AdipoDown }}$ mice when compared to WT mice (Fig. 2G, H and I), while body weight was significantly higher from age 5 weeks to 15 weeks (Fig. 2F). Mice housed individually in metabolic chambers did not show significant changes in food intake, water intake, feces weight and urine volume (Supplementary Fig. 2C, D, E and F).

H\&E staining demonstrated that VEGFB repression induced expansion of gWAT and sWAT (Fig. 2J). Lipid droplets were obviously fused and size increased in BAT of $V e g f b^{A d i p o D o w n}$ mice. Relative cell numbers per microscopic field were significantly decreased by $49.6 \%$ in gWAT, $58.8 \%$ in sWAT and $51.9 \%$ in BAT, and average cell size was increased by 2.0, 2.5 and 2.1 fold correspondingly (Fig. 2K and L). Expression of WAT-specific markers Leptin and Resistin were significantly increased by $74.4 \%$ and $60.7 \%$ in gWAT, $89.4 \%$ and $96.8 \%$ in sWAT, and $63.7 \%$ and $53.2 \%$ in BAT (Fig. 2M and $\mathrm{N}$ ). In contrast, BATspecific genes are all downregulated. Bone morphogenetic protein 7 (Bmp7), a growth factor of TGF family related to BAT development, was significantly decreased by $28.9 \%$ in gWAT, $58.9 \%$ in sWAT and $50.4 \%$ in BAT (Fig. $2 \mathrm{O})$. The cell death-inducing DNA fragmentation factor$\alpha$-like effecter (Cidea), a mitochondria-specific marker of BAT differentiation and thermogenesis (Nishimoto \& Tamori 2017), was decreased by $35.3 \%$ in gWAT, $37.2 \%$ in sWAT and $25.6 \%$ in BAT (Fig. 2P). PRD1-BF1-RIZ1 homologous domain containing 16 (Prdm16), a regulator of thermogenic gene in UCP1-positive adipocytes (Lodhi et al. 2017), was decreased by $41.4 \%$ in gWAT, $38.5 \%$ in sWAT and $36.7 \%$ in BAT (Fig. 2Q). UCP1, a major mitochondrial inner membrane protein responsible for heat generation (Shimizu \& Walsh 2015, Ikeda et al. 2017), was markedly decreased by $28.3 \%$ in gWAT, $52.2 \%$ in sWAT and $41.1 \%$ in BAT at mRNA levels (Fig. 2R) and $89 \%$ in sWAT and $31 \%$ in BAT at protein levels (Fig. 2S and $\mathrm{T}$ ). These results demonstrate that adipose-specific VEGFB repression resulted in dramatic changes in adipose morphology and gene expression toward energy storage.

\section{Adipose-specific VEGFB repression results in reduced energy expenditure}

VEGFB plays important roles in energy metabolism. Vegfb AdipoDown mice had a lower oxygen consumption and lower body temperature (Fig. 3A and B) along with increased body weight (Fig. 3C) and gWAT weight (Fig. 3D). These mice displayed a lower respiratory 


\begin{tabular}{|l|l|l|l|l|}
\hline $\begin{array}{l}\text { Journal of } \\
\text { Endocrinology }\end{array}$ & $Y$ Chen, M Zhao et al. & $\begin{array}{l}\text { VEGFB regulates energy } \\
\text { metabolism }\end{array}$ & $\mathbf{2 4 4 : 3}$ & $\mathbf{5 1 5}$ \\
\hline
\end{tabular}
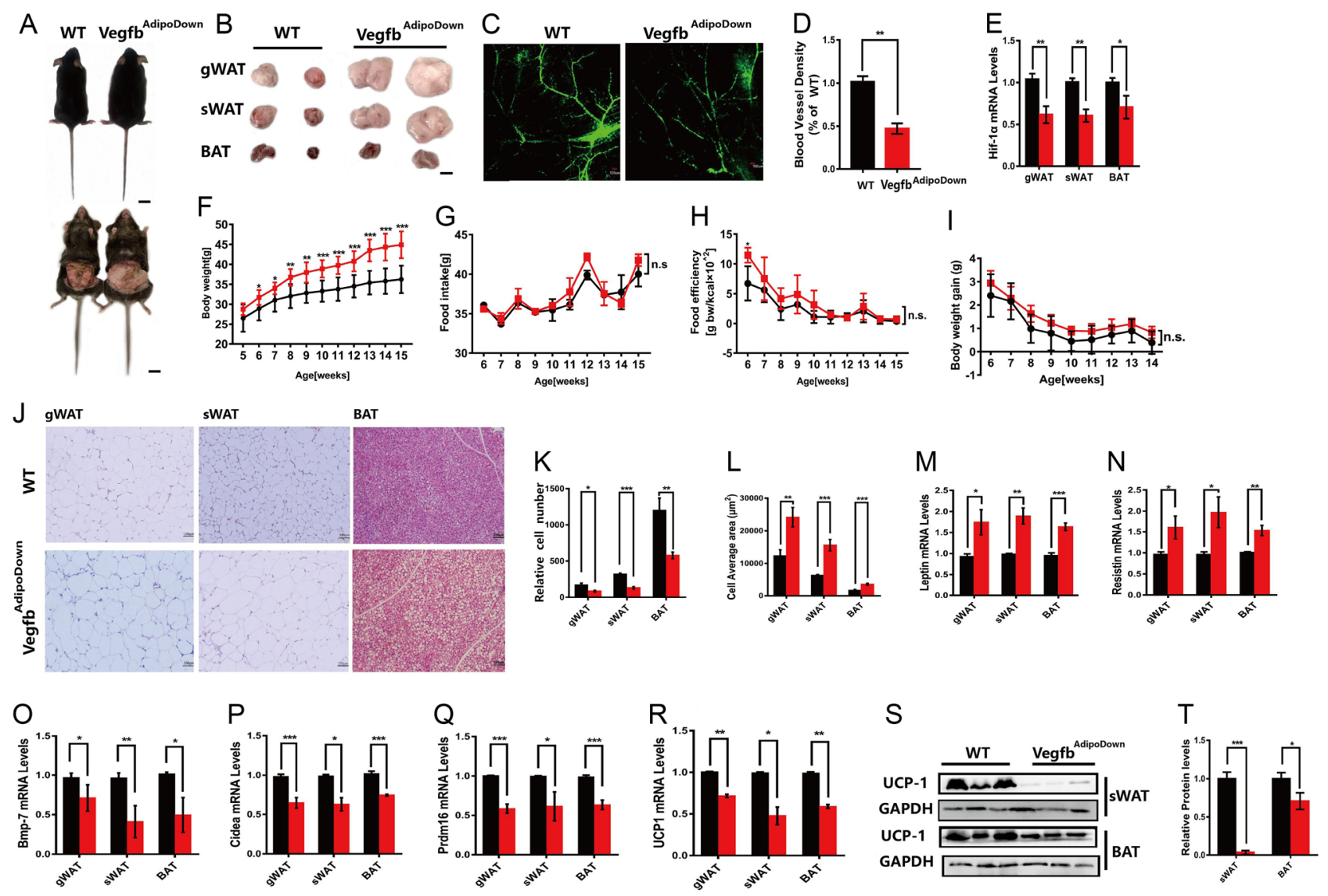

Figure 2

Phenotyping of VegfbAdipoDown mice. (A) Representative macroscopic images of body sizes. (B) Adipose sizes. (C) Confocal images of gWAT after cardiac perfusion of dextran-FITC250000 (scale bar $100 \mu \mathrm{m}, n=3$ ). (D) Quantification of vessel density shown in C. (E) Relative mRNA levels of Hif-1a by qPCR. (F) Average body weight, $(\mathrm{G})$ average food intake, $(\mathrm{H})$ food efficiency and $(\mathrm{I})$ average body weight gains of male mice on regular chow from week 5 to week 15 $(n=8)$. (J) Hematoxylin and eosin (H\&E) staining of adipose tissues. Representative sections of adipose (original magnification 100x, $n=6)$. (K and L) Cell number and average cell area of adipose $\left(\mu \mathrm{m}^{2}\right)$. (M, N, O, P, Q and R) Relative mRNA levels of adipose-associated genes by qPCR, normalized to $18 \mathrm{~S}$ mRNA $(n=3)$. (S) Immunoblotting analysis of UCP-1 $(n=3)$. (T) Quantification of immunoblotting shown in S. VegfbadipoDown bars in red. Data represented by mean \pm S.E.M. n.S., not significant, $* P<0.05, * * P<0.01, * * * P<0.001$.

exchange ratio (RER) as well than their WT littermate (Supplementary Fig. 2G). Body weight and gWAT weight were measured at age of 15 weeks. The average body weight and gWAT weight of VegfbAdipoDown to WT were $42.22 \pm 0.85 \mathrm{~g}$ to $36.51 \pm 1.24 \mathrm{~g}$ (Fig. $3 \mathrm{C}$ ) and $0.86 \pm 0.18 \mathrm{~g}$ to $0.51 \pm 0.12 \mathrm{~g}$ (Fig. 3D) respectively. The gWAT weight rate was significantly increased as well (Fig. 3E). Triglyceride levels were significantly increased by $41.4 \%$ (Fig. 3F). Interestingly, blood glucose level and AUC of IPGTT were not significantly changed and showing a normal glucose response (Fig. 3G and $\mathrm{H}$ ). After intraperitoneal insulin injection, blood glucose level did not change as well. Rate of glucose decay (KITT) was similar to WT, indicating insulin sensitivity was not affected (Fig. 3I and J). Results demonstrated that VegfbadipoDown mice have reduced metabolic and thermogenic activities but preserved glucose sensitivity.

(C) 2020 Society for Endocrinology Published by Bioscientifica Ltd. Printed in Great Britain
The whole-body VEGFB knockout induced adipose whitening and altered gene expression of lipid transporters and glucose transporters (Jin et al. 2018). In this study, adipose-specific VEGFB repression induced expansion and whitening of gWAT, sWAT and BAT as well. Fatp1, Fatp3 and Fatp4 were analyzed and found significantly increased in gWAT and sWAT except Fatp2. Fatp2, Fatp3 and Fatp4 were decreased in BAT except Fatp1 (Fig. 3K, $\mathrm{L}, \mathrm{M}$ and $\mathrm{N}$ ). Results suggest that VEGFB repression may lead to lipid mobilization from energy-consuming BAT to energy-saving WAT. Fatp3 and Fatp4 have no changes in liver, heart and muscle (Supplementary Fig. 2I and J). The oil red $\mathrm{O}$ staining of these tissues also support the results (Supplementary Fig. 2H), indicating that adipose VEGFB reduction does not alter the fatty acid mobilization in muscle and heart. Compare to lipid transporters, glucose transporters were less significantly affected. Glut1 was 

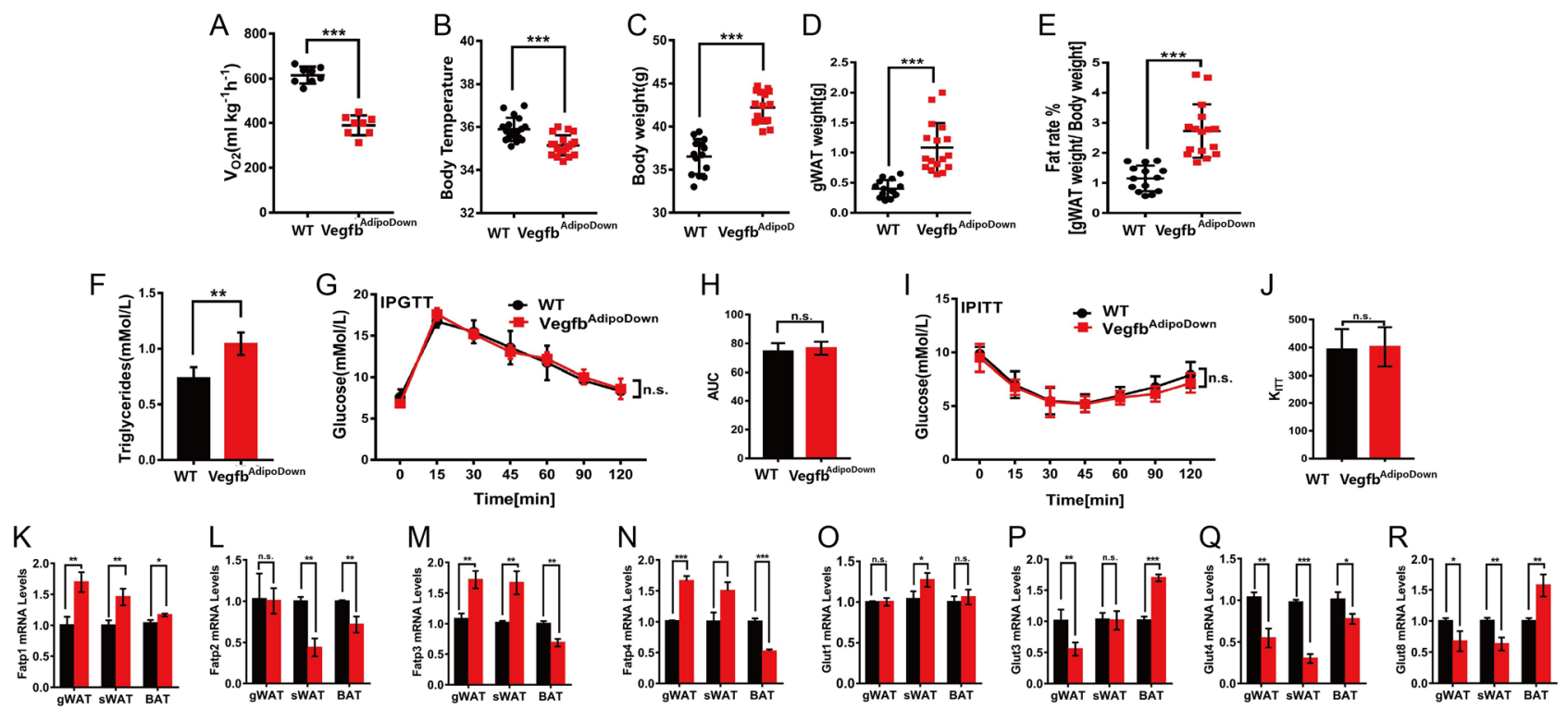

Figure 3

Physiological analysis of VegfbAdipoDown mice. (A) Oxygen consumption measured by $\mathrm{CO}_{2}$ generation. (B) Body temperature. (C) Body weight. (D) gWAT weight. (E) Percentage of adipose tissues weight against body weight. (F) Triglyceride content $(n=6)$. (G and I) IPGTT and IPITT at age of 16 weeks ( $n=6)$. (H) Area under the curve (AUC) of intraperitoneal glucose tolerance test (IPGTT). (J) Rate of glucose decay (KITT) of intraperitoneal insulin tolerance test (IPITT). (K, L, M and N) The mRNA levels of fatty acid transporters (Fatps) $(n=3)$. (O, P, Q and R) The mRNA levels of glucose transporters (Gluts) ( $n=3)$. VegfbAdipoDown bars in red. Data represented by mean \pm S.E.M. n.S., not significant, $* P<0.05, \star \star P<0.01, \star \star \star P<0.001$.

slightly increased in sWAT but not altered in gWAT and BAT (Fig. 3O). Glut3 and Glut8 were upregulated in BAT but downregulated in gWAT (Fig. 3P and R). Glut4 was only downregulated in gWAT. Results indicate glucose transporters may partially account for the altered energy metabolism.

\section{Adipose VEGFB repression leads to insulin resistance on HFD}

Mice were fed on high-fat diet (HFD) and assayed for metabolic changes. VegfbadipoDown mice showed bigger body size than WT mice in the same litters (Fig. 4A). Larger volume of gWAT and sWAT was observed (Fig. 4B). Weight of gWAT, sWAT, BAT and fat rate were dramatically increased in Vegfb AdipoDown mice on HFD (Fig. 4C, D, E and F). VegfbAdipoDown mice had a significantly higher body weight on HFD (Fig. 4G). However, food intake, food efficiency and body weight gain was not significantly changed (Fig. 4H, I and J). Body temperature was lower as expected, although both VegfbadipoDown and WT have slightly higher body temperature than that on regular food (Fig. 4K). Serum triglyceride and cholesterol levels were increased in Vegfb ${ }^{\text {AdipoDown }}$ mice (Fig. $4 \mathrm{~L}$ and M). BAT was surrounded by white adipose tissues in addition to whitening. Consistent whitening and increased lipid droplets accumulation in BAT was observed (Fig. 4N). As expected, cell sizes of gWAT and sWAT were significantly larger than control (Fig. 4N). After 12 weeks of HFD, blood glucose levels were higher and glucose clearance was impaired in IPGTT. This is also reflected by the high AUC values (Fig. $4 \mathrm{O}$ and P). After intraperitoneal insulin injection, blood glucose level was higher from $15 \mathrm{~min}$ to $120 \mathrm{~min}$ (Fig. 4Q). The rate of glucose decay (KITT) is lower than WT mice (Fig. 4R), indicating that Vegfb $b^{\text {AdipoDown }}$ mice became insensitive to insulin. Consistently, VEGFB repression decreased expression of Bmp7, Prdm16 and UCP-1, and increased expression of leptin (Fig. 4S, T, U and $\mathrm{V}$ ). These results suggest that adipose-specific VEGFB repression can increase risk of diabetic development on high-fat diet.

\section{VEGFB repression has broad effect on cell metabolism}

RNA-Seq was used to investigate genome-wide effect of VEGFB repression. A total of 1579 DEGs (233 up and 1346 down) in WAT and 654 DEGs (282 up and 372 down) in BAT were identified. There are 1374 DEGs changed in gWAT only, 449 DEGs BAT only and 205 DEGs in both gWAT and BAT (Fig. 5A and B). KEGG pathway analysis showed that more diversified genes are involved in biological processes, including metabolism, 


\begin{tabular}{l|l|l|r|r|}
$\begin{array}{l}\text { Journal of } \\
\text { Endocrinology }\end{array}$ & $Y$ Chen, M Zhao et al. & $\begin{array}{l}\text { VEGFB regulates energy } \\
\text { metabolism }\end{array}$ & $\mathbf{2 4 4 : 3}$ & $\mathbf{5 1 7}$ \\
\hline
\end{tabular}
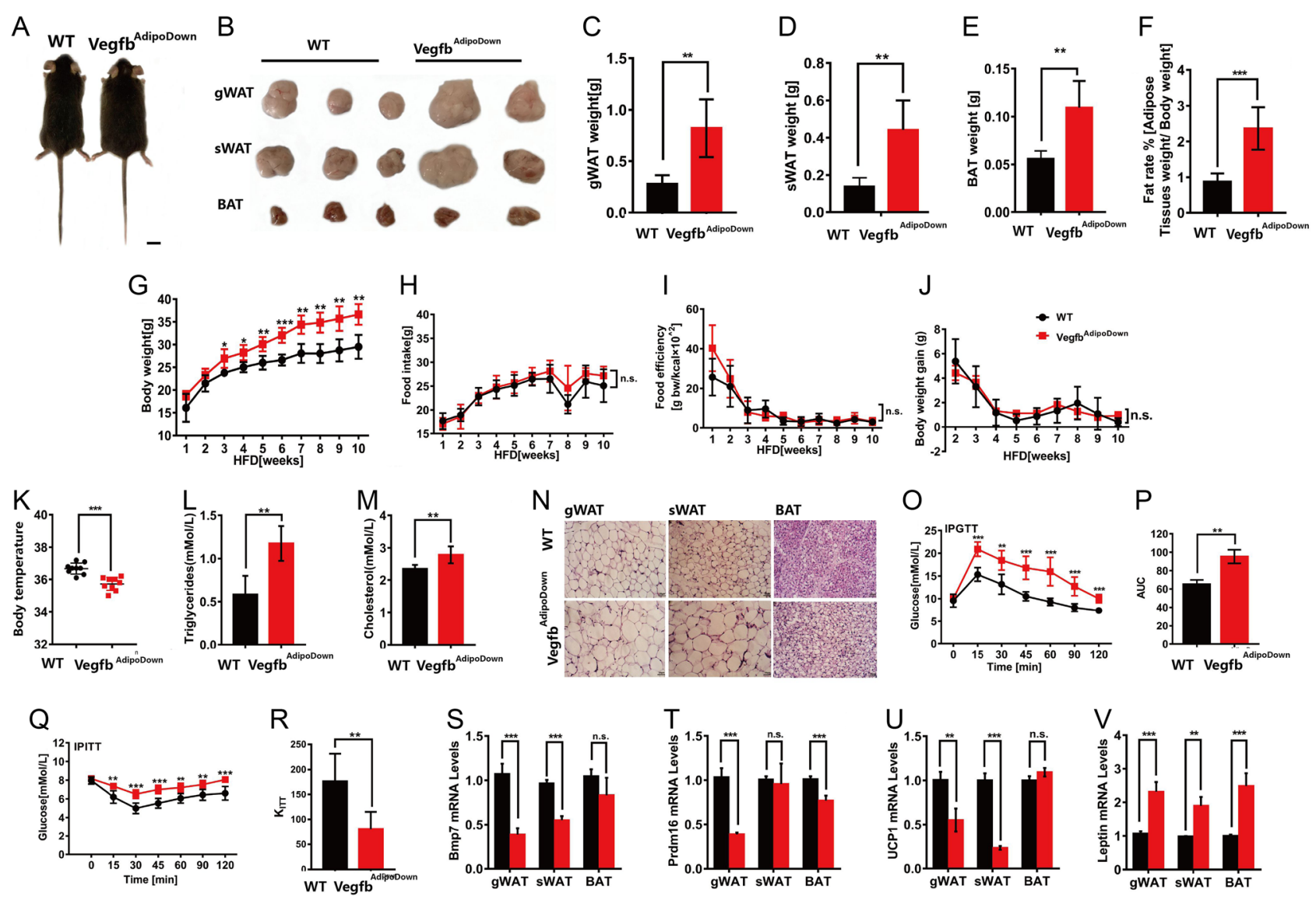

\section{Figure 4}

Phenotyping of VegfbAdipoDown mice on HFD. Representative macroscopic images of body sizes (A) and adipose sizes (B). (C) gWAT weight. (D) sWAT weight. (E) BAT weight. (F) Percentage of adipose tissues weight against body weight. Body weight gains (G), food intake (H), food efficiency (I) and body weight gains (U) of male mice on HFD from week 3 to week $12(n=6)$. (K) Body temperature. (L) Triglyceride content. (M) Serum cholesterol levels $(n=5)$. (N) H\&E staining of adipose tissues. Representative sections of adipose (original magnification 100x, $n=3$ ). (O) IPGTT and (Q) IPITT at age of 14 months ( $n=7$ ). (P) Area under the curve (AUC) of IPGTT. (R) Rate of glucose decay (KITT) in IPITT. (S, T, U and V) Relative mRNA levels of adipose-associated genes by qPCR, normalized to $18 \mathrm{~S}$ mRNA $(n=3)$. VegfbAdipoDown bars in red. Data represented by mean \pm S.E.M. n.S., not significant, $* P<0.05, * \star P<0.01, * \star \star P<0.001$.

cellular process, environmental information processing, genetic information processing and organismal system (Fig. 5C and D). Many metabolism-related genes are differentially regulated under VEGFB (Fig. 6A, B, C, D, E and F). For example, epididymal-specific glutathione peroxidase 5 (GPX5), which is an important antioxidant and can protect cells from oxidative stress (Taylor et al. 2013), was dramatically increased in gWAT and decreased in BAT. Pyridoxal phosphatase (PDXP) that regulates expression of vitamin B6 (VB6) (Li et al. 2019) was decreased in gWAT and increased in BAT. Many mitochondrial genes were changed under VEGFB (Supplementary Fig. 1A). For example, ATP synthases were obviously decreased in gWAT and BAT (Supplementary Fig. 1A). Results demonstrate that broad range of genes involved in metabolism are changed under VEGFB.

\section{Discussion}

A new mouse model of adipose-specific VEGFB repression was generated using CRISPR-dCas9 technology to investigate VEGFB-regulated adipose development and function. Jin et al. generated Vegf $\mathrm{b}^{-/-}$mice using CRISPR-Cas9 system and found that VEGFB deletion induces obese phenotype (Jin et al. 2018), similar to the study of Hagberg's Vegf $b_{-/}^{-}$mice. But contribution of adipose-expressed VEGFB is not studied. Vegfb ${ }^{\text {AdipoDown }}$ mice showed larger body weight, body size and WAT expansion (Fig. 2A, B and F). Adipose-specific VEGFB repression induces morphologic and functional transformation of adipose tissues toward WAT development (Figs 2J and $4 \mathrm{~N})$, accompanied with upregulation of WAT-associated genes and downregulation of BAT-associated genes (Figs $2 \mathrm{M}, \mathrm{N}, \mathrm{O}, \mathrm{P}, \mathrm{Q}, \mathrm{R}$ and $4 \mathrm{~S}, \mathrm{~T}, \mathrm{U}, \mathrm{V})$. In accordance with 
A

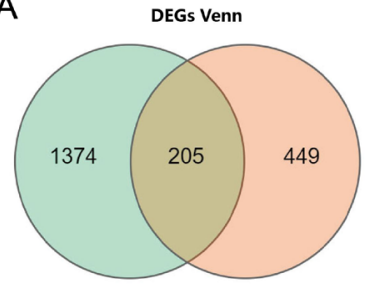

C

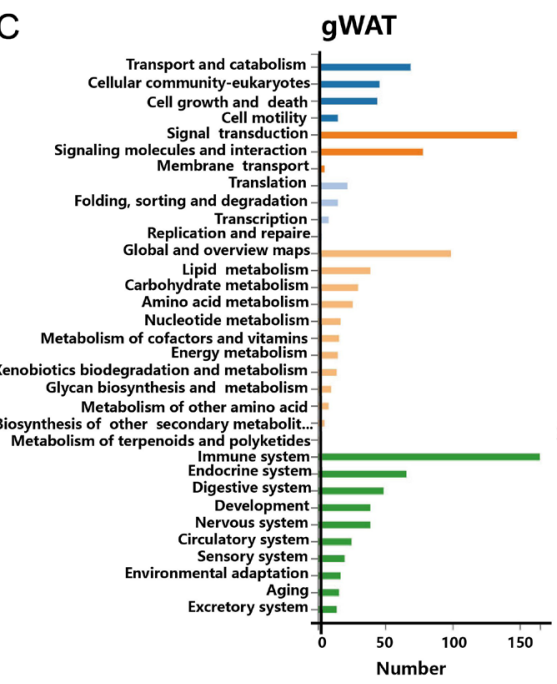

B

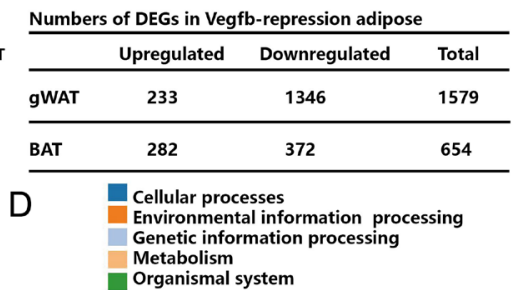

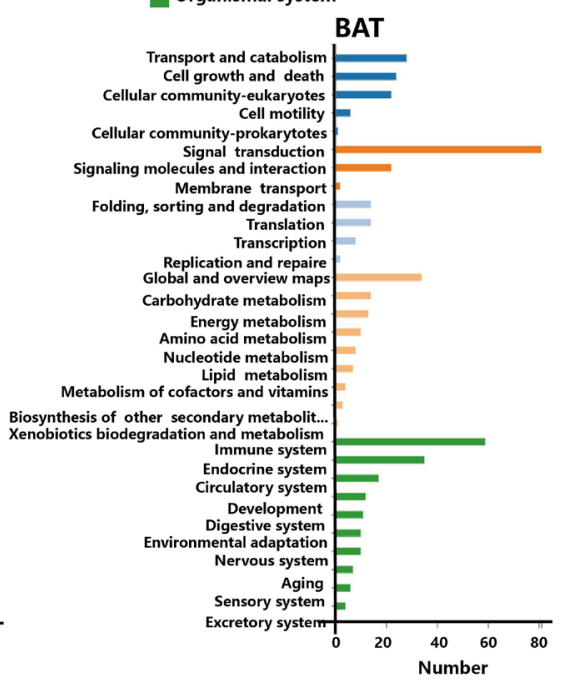

Figure 5

KEGG analysis of DEGs. (A) Illustration of DEGs in Venn. (B) Numbers of DEGs of Vegfb-repressed to WT adipose tissues. KEGG pathway analysis of gWAT (C) and BAT (D). DEGs were selected based on expression levels $\geq 10$ by fragments per kilobase of transcript per million $\geq 10$, fold change $\geq 2$ (lg2 $\geq 1)$ and $Q$ value $<0.05$.
BAT whitening, there is a reduced respiratory rate and low body temperature in Vegfb ${ }^{\text {AdipoDown }}$ mice (Fig. 3A and B). All the phenotypes basically recapitulated the ones in the universal VEGFB-knockout mouse model except insulin sensitivity in HFD condition (Fig. 4O, P, Q and R).

On regular diet, insulin sensitivity and glucose clearance are not affected by adipose VEGFB repression (Fig. 3G and I). Some studies have shown that intracellular fatty acid accumulation can directly impair insulin signaling and glucose uptake, causing insulin resistance (Schmitz-Peiffer et al. 1997, Boström et al. 2007, SchmitzPeiffer 2010). It is seemingly healthy for adipose-specific VEGFB-knockdown mice. Vegf $b^{\text {AdipoDown }}$ mice have similar food efficiency as WT mice (Fig. 2H). However, Vegfb $b^{\text {AdipoDown }}$ mice cannot take the high fat load (Fig. 4O, $\mathrm{P}, \mathrm{Q}$ and $\mathrm{R})$.

FATPs are responsible for fatty acid transport to adipose tissues (Schaffer \& Lodish 1994, Hirsch et al. 1998, Kazantzis \& Stahl 2012). Significant increase of Fatp1, Fatp3 and Fatp4 mRNA levels in adipose tissues supports the role of VEGFB on fatty acid mobilization toward WAT (Fig. 3K, L, M and N). Hagberg et al. found that VEGFB deletion leads to downregulation of FATP3 and FATP4 in endothelial cells of muscle, heart and BAT. This reduced uptake leads to excessive accumulation of lipids in WAT (Hagberg et al. 2010). We have found that increase of FATP3 and FATP4 expression in WAT help to mobilize the lipids toward storage as well (Jin et al. 2018). However, the high serum triglyceride may reflect an inefficient removal of lipid by FATP3 and FATP4 in white adipose tissues on HFD. In our previous study, Vegfb heterozygous deletion reduced $V e g f b$ mRNA levels by up to $90 \%$ in mitochondriarich tissues including heart, skeletal muscle and BAT (Jin et al. 2018). This result supports that mitochondriarich tissues rely on fatty acids as main source for energy production and heat generation (Hagberg et al. 2010). Relatively passive WAT cannot efficiently handle the surplus lipid on HFD.

High-throughput RNA-Seq showed that not only VEGFB repression in adipose tissue affect lipid metabolism, but also metabolism of carbohydrate, amino acid, nucleotides, cofactors and vitamins (Fig. 6). These results confirm the important regulatory roles of VEGFB on broad aspect of metabolism. Robciuc and Cao respectively revealed that VEGFB/VEGFR1 acts as a decoy pathway for VEGFA/VEGFR2. Overexpressing VEGFB or tissue-specific loss of VEGFR1 on endothelial cells results in vascular expansion and improves metabolic outcome induced by HFD (Robciuc et al. 2016, Seki et al. 2018). This is consistent with our results that interaction between VEGFB/VEGFR1 and VEGF/VEGFR2 signaling is likely involved. When VEGFB was decreased, VEGFR1 binds more VEGF and inhibits VEGF/VEGFR2 pathway. 
A
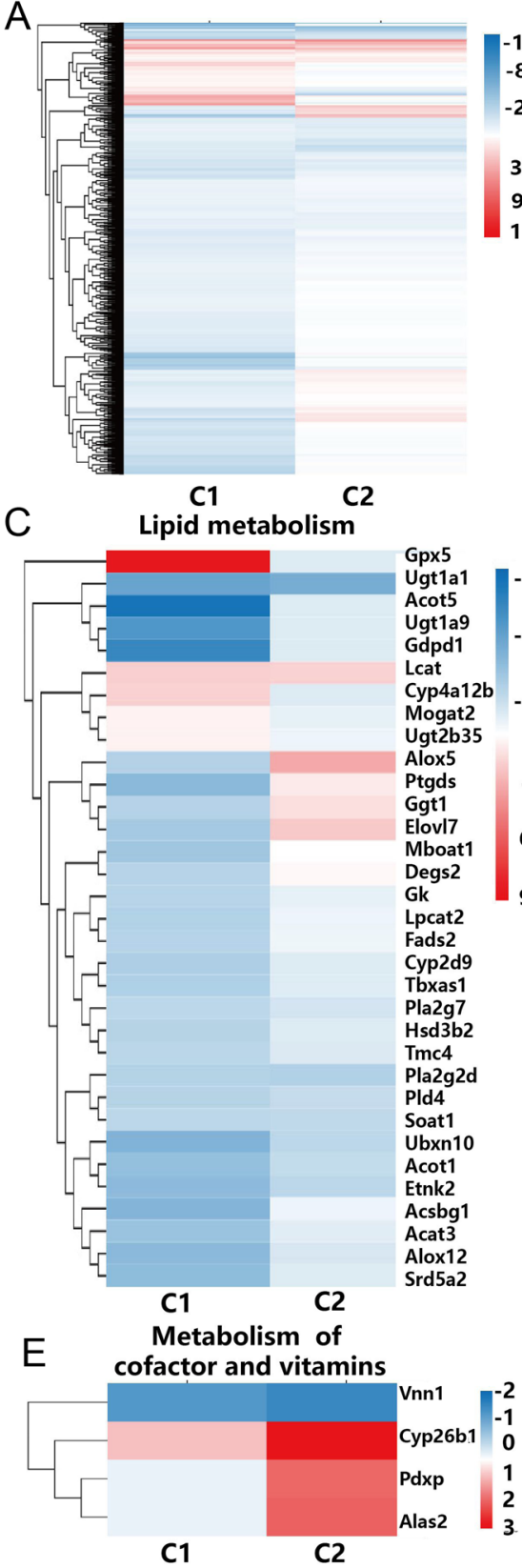

B

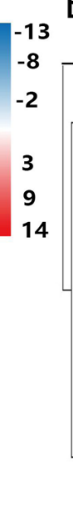

Carbohydrate metabolism

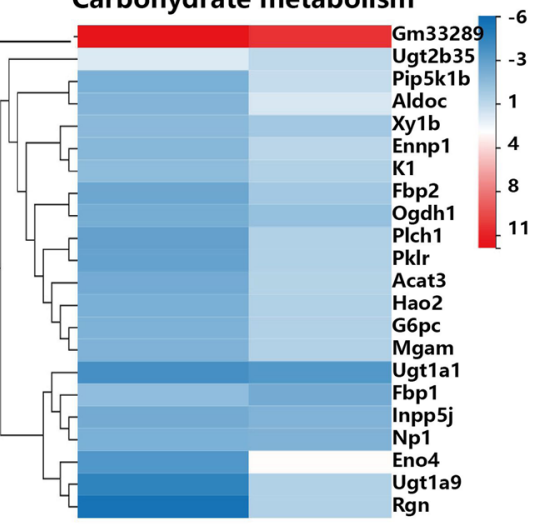

C1

C2

\section{Amino acid metabolism}

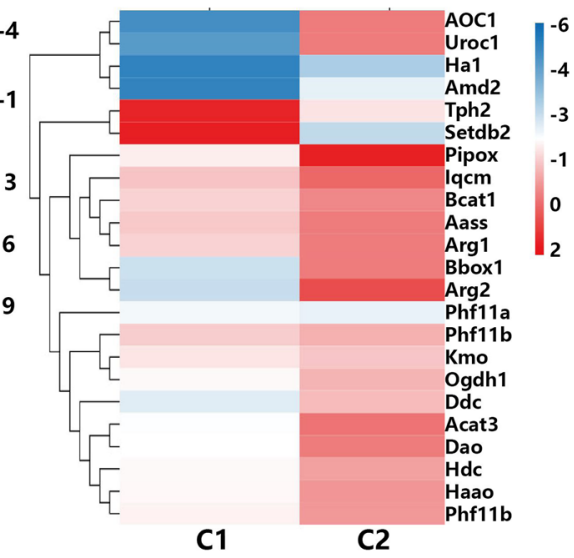

F Nucleotide metabolism

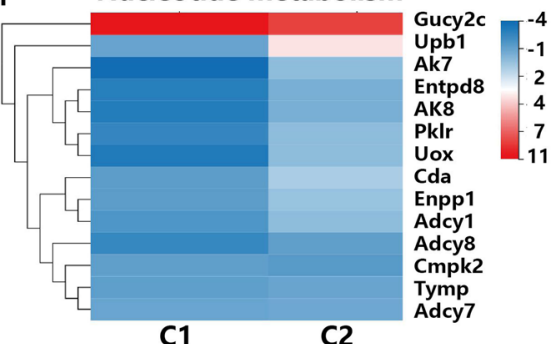

C1: $\log 2(\mathrm{Tg}-\mathrm{gWAT} / \mathrm{WT}-\mathrm{gWAT})$ C2: $\log 2(T g-B A T / W T-B A T)$

\section{Figure 6}

DEGs analysis of metabolism related genes. (A) Heat map of gWAT (C1) and BAT DEGs (C2) from VegfbAdipoDown against wild type mice. (B) Heat map of carbohydrate metabolism. (C) Heat map of lipid metabolism. (D) Heat map of amino acid metabolism. (E) Heat map of metabolism of cofactors and vitamins. (F) Heat map of nucleotides metabolism. Gene expression level cutoff: fragments per kilobase of transcript per million $\geq 10$ and $Q$ value $<0.05$.
Studies have been focused on vasculature of adipose tissues considering VEGFB's roles on development and metabolism. However, it is clear beyond the role of vasculature. Adipose tissue adapts to its environment through two processes. One is quick response by shuffling fatty acid from energy storage (white adipose) to energy generation (heart, muscle and BAT). Another is long-term adaption by adjusting ratio of tissues that stores energy (white adipose) and tissues that consumes energy (heart, muscle and BAT). Although it is a prerequisite for adipose to have proper amount of vasculature for these functional demand, VEGFB is highly expressed in mitochondria-rich tissue and has minimal effect on vasculature development. Its major role is regulating the balance of adipose development and energy metabolism with VEGFA. As far as we know, VEGFR2 is not expressed on adipocytes and VEGFR1 does not generate downstream signals. Cao's studies on mouse and zebrafish suggest that VEGFB function through Nrp1, independent of VEGFR1 (Jensen et al. 2015). This is consistent to our study that VEGFR1 and Nrp1 are oppositely regulated by VEGFB in adipocytes (Fig. 1D).

In conclusion, adipose VEGFB regulates adipose development and function through up and down 
regulating wide range of genes from adipogenesis to metabolism. Approximately 50\% VEGFB repression in adipose tissues (gWAT, sWAT and BAT) can almost completely recapitulate the effect of whole body VEGFB deletion (Supplementary Fig. 1D). All the results confirm that adipose-expressed VEGFB is a major regulator of adipose development and functions and plays indispensable roles in metabolism.

\section{Supplementary materials}

This is linked to the online version of the paper at https://doi.org/10.1530/ JOE-19-0341.

\section{Declaration of interest}

The authors declare that there is no conflict of interest that could be perceived as prejudicing the impartiality of the research reported.

\section{Funding}

This work is supported in part by National Natural Science Foundation of China (81270953) and Natural Science Foundation of Jilin Province (20160101344JC). The funders had no roles in study design, data collection and analysis or decision to publish.

\section{Author contribution statement}

$Y W Z, Y C, X C F$ and $L Q Z$ conceived and designed the experiments. $Y C$, M Y Z, C H W, Y T Z, T T Z, H Z W, M J Z, S A, M W G and H Y C carried out experiments. $Y C, M Y Z$ and $M X L$ analyzed the data. $Y C$ and $D L$ contributed reagents/materials/analysis tools. $Y W Z$ and $Y C$ wrote the paper. $Y W Z, Y$ $C$ and $X D$ L edited and revised manuscript; $Y$ W Z approved final version of manuscript. All authors were involved in revising the manuscript and had final approval of the submitted and published versions.

\section{Acknowledgments}

The authors thank Huiyan Wu for microinjection and mouse colony maintenance.

\section{References}

Boström P, Andersson L, Rutberg M, Perman J, Lidberg U, Johansson BR, Fernandez-Rodriguez J, Ericson J, Nilsson T, Borén J, et al. 2007 SNARE proteins mediate fusion between cytosolic lipid droplets and are implicated in insulin sensitivity. Nature Cell Biology 9 1286-1293. (https://doi.org/10.1038/ncb1648)

Cai L, Lubitz J, Flegal KM \& Pamuk ER 2010 The predicted effects of chronic obesity in middle age on medicare costs and mortality. Medical Care 48 510-517. (https://doi.org/10.1097/ MLR.0b013e3181dbdb20)

Castro AV, Kolka CM, Kim SP \& Bergman RN 2014 Obesity, insulin resistance and comorbidities-mechanisms of association. Arquivos
Brasileiros de Endocrinologia e Metabologia 58 600-609. (https://doi. org/10.1590/0004-2730000003223)

Cedikova M, Kripnerová M, Dvorakova J, Pitule P, Grundmanova M, Babuska V, Mullerova D \& Kuncova J 2016 Mitochondria in white, brown, and beige adipocytes. Stem Cells International 2016 1-11. (https://doi.org/10.1155/2016/6067349)

Chen Y, Zhao M, Zheng T, Adlat S, Jin H, Wang C, Li D, Zaw Myint MZ, Yao Y, Xu L, et al. 2019 Repression of adipose vascular endothelial growth factor reduces obesity through adipose browning. American Journal of Physiology: Endocrinology and Metabolism 316 E145-E155. (https://doi.org/10.1152/ajpendo.00196.2018)

Cinti S 2008 Reversible transdifferentiation in the adipose organ. International Journal of Pediatric Obesity 3 (Supplement 2) 21-26. (https://doi.org/10.1080/17477160802404665)

De Matteis R, Lucertini F, Guescini M, Polidori E, Zeppa S, Stocchi V, Cinti S \& Cuppini R 2013 Exercise as a new physiological stimulus for brown adipose tissue activity. Nutrition, Metabolism, and Cardiovascular Diseases 23 582-590. (https://doi.org/10.1016/j. numecd.2012.01.013)

Duan J, Lu G, Hong Y, Hu Q, Mai X, Guo J, Si X, Wang F \& Zhang Y 2018 Live imaging and tracking of genome regions in crispr/dcas9 knock-in mice. Genome Biology 19 192. (https://doi.org/10.1186/s13059-0181530-1)

Enerbäck S, Jacobsson A, Simpson EM, Guerra C, Yamashita H, Harper ME \& Kozak LP 1997 Mice lacking mitochondrial uncoupling protein are cold-sensitive but not obese. Nature $\mathbf{3 8 7} 90-94$. (https://doi. org/10.1038/387090a0)

Esteve Ràfols M 2014 Adipose tissue: cell heterogeneity and functional diversity. Endocrinologia y Nutricion 61 100-112. (https://doi. org/10.1016/j.endonu.2013.03.011)

Fain JN, Madan AK, Hiler ML, Cheema P \& Bahouth SW 2004 Comparison of the release of adipokines by adipose tissue, adipose tissue matrix, and adipocytes from visceral and subcutaneous abdominal adipose tissues of obese humans. Endocrinology 145 2273-2282. (https://doi.org/10.1210/en.2003-1336)

Hagberg CE, Falkevall A, Wang X, Larsson E, Huusko J, Nilsson I, van Meeteren LA, Samen E, Lu L, Vanwildemeersch M, et al. 2010 Vascular endothelial growth factor B controls endothelial fatty acid uptake. Nature 464 917-921. (https://doi.org/10.1038/nature08945)

Hirsch D, Stahl A \& Lodish HF 1998 A family of fatty acid transporters conserved from mycobacterium to man. PNAS 95 8625-8629. (https://doi.org/10.1073/pnas.95.15.8625)

Ikeda K, Kang Q, Yoneshiro T, Camporez JP, Maki H, Homma M, Shinoda K, Chen Y, Lu X, Maretich P, et al. 2017 Ucp1-independent signaling involving serca2b-mediated calcium cycling regulates beige fat thermogenesis and systemic glucose homeostasis. Nature Medicine 23 1454-1465. (https://doi.org/10.1038/nm.4429)

James WP 2008 The epidemiology of obesity: the size of the problem. Journal of Internal Medicine 263 336-352. (https://doi.org/10.1111/ j.1365-2796.2008.01922.x

Jensen LD, Nakamura M, Bräutigam L, Li X, Liu Y, Samani NJ \& Cao Y 2015 Vegf-b-neuropilin-1 signaling is spatiotemporally indispensable for vascular and neuronal development in zebrafish. PNAS 112 E5944-E5953. (https://doi.org/10.1073/pnas.1510245112)

Jin H, Li D, Wang X, Jia J, Chen Y, Yao Y, Zhao C, Lu X, Zhang S, Togo J, et al. 2018 Vegf and vegfb play balancing roles in adipose differentiation, gene expression, and function. Endocrinology 159 2036-2049. (https://doi.org/10.1210/en.2017-03246)

Karpanen T, Bry M, Ollila HM, Seppänen-Laakso T, Liimatta E, Leskinen H, Kivelä R, Helkamaa T, Merentie M, Jeltsch M, et al. 2008 Overexpression of vegf-b in mouse heart alters cardiac lipid metabolism and induces myocardial hypertrophy. Circulation Research 103 1018-1026. (https://doi.org/10.1161/CIRCRESAHA.108.178459)

Kazantzis M \& Stahl A 2012 Fatty acid transport proteins, implications in physiology and disease. Biochimica and Biophysica Acta 1821 852-857. (https://doi.org/10.1016/j.bbalip.2011.09.010) 
Kershaw EE \& Flier JS 2004 Adipose tissue as an endocrine organ. Journal of Clinical Endocrinology and Metabolism 89 2548-2556. (https://doi. org/10.1210/jc.2004-0395)

Kopelman PG 2000 Obesity as a medical problem. Nature $\mathbf{4 0 4} 635-643$. (https://doi.org/10.1038/35007508)

Kreuger J, Claesson-Welsh L, Olsson AK \& Dimberg A 2006 Vegf receptor signalling - in control of vascular function. Nature Reviews Molecular Cell Biology 7 359-371.

Li H, Wang Y, Yang H, Liu L, Wang J \& Zheng N 2019 Lactoferrin induces the synthesis of vitamin B6 and protects HUVEC functions by activating PDXP and the PI3K/AKT/ERK1/2 pathway. International Journal of Molecular Sciences 20 E587. (https://doi.org/10.3390/ ijms20030587)

Lodhi IJ, Dean JM, He A, Park H, Tan M, Feng C, Song H, Hsu FF \& Semenkovich CF 2017 Pexrap inhibits prdm16-mediated thermogenic gene expression. Cell Reports 20 2766-2774. (https://doi. org/10.1016/j.celrep.2017.08.077)

Luna-Luna M, Medina-Urrutia A, Vargas-Alarcón G, Coss-Rovirosa F, Vargas-Barrón J \& Pérez-Méndez Ó 2015 Adipose tissue in metabolic syndrome: onset and progression of atherosclerosis. Archives of Medical Research 46 392-407. (https://doi.org/10.1016/j. arcmed.2015.05.007)

Madsen L, Pedersen LM, Lillefosse HH, Fjaere E, Bronstad I, Hao Q, Petersen RK, Hallenborg P, Ma T, De Matteis R, et al. 2010 Ucp1 induction during recruitment of brown adipocytes in white adipose tissue is dependent on cyclooxygenase activity. PLOS ONE $\mathbf{5}$ e11391. (https://doi.org/10.1371/journal.pone.0011391)

Malumbres M, Mangues R, Ferrer N, Lu S \& Pellicer A 1997 Isolation of high molecular weight dna for reliable genotyping of transgenic mice. BioTechniques 22 1114-1119. (https://doi.org/10.2144/97226st03)

Mick GJ, Wang X \& Mccormick K 2002 White adipocyte vascular endothelial growth factor: regulation by insulin. Endocrinology 143 948-953. (https://doi.org/10.1210/endo.143.3.8673)

Mission JF, Marshall NE. \& Caughey AB 2015 Pregnancy risks associated with obesity. Obstetrics and Gynecology Clinics of North America 42 335-353. (https://doi.org/10.1016/j.ogc.2015.01.008)

Nishimoto Y \& Tamori Y 2017 Cide family-mediated unique lipid droplet morphology in white adipose tissue and brown adipose tissue determines the adipocyte energy metabolism. Journal of Atherosclerosis and Thrombosis 24 989-998. (https://doi.org/10.5551/jat.RV17011)

Qi LS, Larson MH, Gilbert LA, Doudna JA, Weissman JS, Arkin AP \& Lim WA 2013 Repurposing Crispr as an rna-guided platform for sequence-specific control of gene expression. Cell 152 1173-1183. (https://doi.org/10.1016/j.cell.2013.02.022)

Richard D \& Picard F 2011 Brown fat biology and thermogenesis. Frontiers in Bioscience 16 1233-1260. (https://doi.org/10.2741/3786)

Richard D, Carpentier AC, Doré G, Ouellet V \& Picard F 2010 Determinants of brown adipocyte development and thermogenesis.
International Journal of Obesity 34 (Supplement 2) S59-S66. (https:// doi.org/10.1038/ijo.2010.241)

Robciuc MR, Kivelä R, Williams IM, de Boer JF, van Dijk TH, Elamaa H, Tigistu-Sahle F, Molotkov D, Leppänen VM, Käkelä R, et al. 2016 Vegfb/vegfr1-induced expansion of adipose vasculature counteracts obesity and related metabolic complications. Cell Metabolism 23 712-724. (https://doi.org/10.1016/j.cmet.2016.03.004)

Rodeheffer MS, Birsoy K \& Friedman JM 2008 Identification of white adipocyte progenitor cells in vivo. Cell 135 240-249. (https://doi. org/10.1016/j.cell.2008.09.036)

Schaffer JE \& Lodish HF 1994 Expression cloning and characterization of a novel adipocyte long chain fatty acid transport protein. Cell $\mathbf{7 9}$ 427-436. (https://doi.org/10.1016/0092-8674(94)90252-6)

Schmitz-Peiffer C 2010 Targeting ceramide synthesis to reverse insulin resistance. Diabetes 59 2351-2353. (https://doi.org/10.2337/db100912)

Schmitz-Peiffer C, Browne CL, Oakes ND, Watkinson A, Chisholm DJ, Kraegen EW \& Biden TJ 1997 Alterations in the expression and cellular localization of protein kinase $\mathrm{C}$ isozymes epsilon and theta are associated with insulin resistance in skeletal muscle of the high-fat-fed rat. Diabetes 46 169-178. (https://doi.org/10.2337/ diab.46.2.169)

Seki T, Hosaka K, Fischer C, Lim S, Andersson P, Abe M, Iwamoto H, Gao Y, Wang X, Fong GH, et al. 2018 Ablation of endothelial vegfr 1 improves metabolic dysfunction by inducing adipose tissue browning. Journal of Experimental Medicine 215 611-626. (https://doi. org/10.1084/jem.20171012)

Shimizu I \& Walsh K 2015 The whitening of brown fat and its implications for weight management in obesity. Current Obesity Reports 4 224-229. (https://doi.org/10.1007/s13679-015-0157-8)

Stuttfeld E \& Ballmer-Hofer K 2009 Structure and function of VEGF receptors. IUBMB Life 61 915-922. (https://doi.org/10.1002/iub.234)

Sun C, Zeng R, Cao G, Song Z, Zhang Y \& Liu C 2015 Vibration training triggers brown adipocyte relative protein expression in rat white adipose tissue. BioMed Research International 2015 919401. (https:// doi.org/10.1155/2015/919401)

Taylor A, Robson A, Houghton BC, Jepson CA, Ford WC \& Frayne J 2013 Epididymal specific, selenium-independent GPX5 protects cells from oxidative stress-induced lipid peroxidation and DNA mutation. Human Reproduction 28 2332-2342. (https://doi.org/10.1093/humrep/ $\operatorname{det} 237)$

Wang QA, Tao C, Gupta RK \& Scherer PE 2013 Tracking adipogenesis during white adipose tissue development, expansion and regeneration. Nature Medicine 19 1338-1344.

WHO Expert Consultation 2004 Appropriate body-mass index for Asian populations and its implications for policy and intervention strategies. Lancet 363 157-163. (https://doi.org/10.1016/S01406736(03)15268-3)

Received in final form 4 December 2019

Accepted 3 January 2020

Accepted Manuscript published online 7 January 2020 https://joe.bioscientifica.com https://doi.org/10.1530/JOE-19-0341
(C) 2020 Society for Endocrinology Published by Bioscientifica Ltd. Printed in Great Britain 